\title{
Applications of Titania Atomic Layer Deposition in the Biomedical Field and Recent Updates
}

\author{
Mina Shahmohammadi ${ }^{1}$, Bin Yang ${ }^{2 *}$ and Christos G Takoudis ${ }^{1,3 *}$ \\ ${ }^{1}$ Department of Chemical Engineering, University of Illinois at Chicago, USA \\ ${ }^{2}$ Department of Restorative Dentistry, University of Illinois at Chicago, USA \\ ${ }^{3}$ Department of Bioengineering, University of Illinois at Chicago, USA
}

*Corresponding author: Bin Yang, Department of Restorative Dentistry, University of Illinois at Chicago, 801 S Paulina St, Chicago, IL 60612, USA, Email: yangbin@uic.edu

Christos G. Takoudis, Department of Chemical Engineering and Bioengineering, University of Illinois at Chicago, 851 S. Morgan St. Chicago, IL 60607, USA, Email: takoudis@uic.edu

To Cite This Article: Mina Shahmohammadi, Bin Yang, Christos G Takoudis. Applications of Titania Atomic Layer Deposition in the Biomedical Field and Recent Updates. 2020 - 8(6). AJBSR.MS.ID.001321. DOI: 10.34297/AJBSR.2020.08.001321.

Received: 㘹 April 24, 2020; Published: 㘹 May 05, 2020

\begin{abstract}
Atomic Layer Deposition (ALD) is a promising technique to precisely deposit nanometer-thick films on inorganic and organic substrates. ALD is known for the deposition of uniform, conformal, and pinhole-free thin films with a controllable thickness with sequential and self-limiting gaseous reactions on the substrate surface of interest. ALD thin films have a wide range of applications, from semiconductor industries to medical devices. In many studies, ALD thin films are used to modify the biomaterial surface properties for particular biomedical applications. Here, polymethyl methacrylate, titanium-based alloys, and collagen were chosen as substrates for this review; thanks to their biocompatibility, these substrates have several applications in the dental, medical, and biomedical fields. The ALD of Titanium (IV) oxide $\left(\mathrm{TiO}_{2}\right)$ films has been selected for this review as a biocompatible material which has attracted increasing attention in biomedical applications. Selected publications focusing on the modification of the surface of these biomaterials with $\mathrm{TiO}_{2} \mathrm{ALD}$ are discussed.
\end{abstract}

Keywords: Atomic Layer Deposition, Surface Functionalization, Titanium Oxide, Polymethyl methacrylate, Ti-based implants, collagen

\section{Introduction}

Atomic layer deposition (ALD) is a vapor-phase technique to deposit thin films of metals, oxides, sulfides, nitrides, and metalorganic frameworks (MOF) on various substrates [1-3]. The films produced by ALD are known to be uniform across the substrate, conformal, and pinhole-free [4]. ALD is capable of depositing films with controllable thickness even on complex 3-D substrates as well as substrates with high aspect ratio [5]. There are three types of ALD: Thermal ALD, Plasma Enhanced ALD (PE-ALD), and Radical Enhanced ALD (RE-ALD) [2,5]. Each one of these has advantages and disadvantages, which depend on the materials involved, deposition conditions, and desired applications [2,5]. Thermal ALD occurs through surface chemical reactions, while in PE-ALD, the substrate is exposed to plasma discharge, energetic ions, and bombarded electrons; RE-ALD is a process similar to PE-ALD except that the substrate is exposed to radicals [5]. PE-ALD and RE-ALD are preferable for heat-sensitive biomaterials because they can be performed at lower temperatures compared to thermal ALD; however, thermal ALD would generally result in higher conformity and there is no likely damage to the substrates during plasma pulses as it is plausible in PE-ALD [3]. More detailed information on each type of ALD can be found in earlier review papers [2-5]. ALD is a cyclic process composed of four steps (Figure 1). During the first step, the precursor, which is usually a metal surrounded with organic functional groups, is introduced into the reactor and chemisorbed on the substrate. The second step is purging unreacted precursor molecules out of the reaction chamber with an inert gas such as $\mathrm{N}_{2}$, Ar, etc. Then the oxidizer/co-reactant (e.g., $\mathrm{O}_{2}, \mathrm{O}_{3}$, water vapor, $\mathrm{O}_{2}$ plasma) is pulsed into the reactor and reacts with the precursor on the substrate surface. The last step is purging unreacted oxidizer molecules and surface reaction byproducts with the inert gas out of the reaction chamber. After each cycle, a layer of atomically thick of the desired material is produced 
on the surface. These four steps are repeated cyclically until the desired nanometer length scale film thickness is achieved. Thus, ALD is promising regarding precise control of the film thickness with atomic thickness precision. Recently, ALD has attracted much attention to modify and functionalize the surface of biomaterials and biomedical implants due to its sequential exposure to the reactants which allows conformal deposition of inorganic materials onto organic and metal substrates. Specifically, titanium(IV) dioxide $\left(\mathrm{TiO}_{2}\right)$ has been widely used thanks to its biocompatibility, antimicrobial activity, hydrophilicity, excellent corrosion resistance, aesthetics, and low cost $[6,7]$. Moreover, $\mathrm{TiO}_{2}$ is an important biomedical material due to its nontoxicity nature in contact with the human body $[8,9]$ for these reasons, some of the recent studies which used ALD to deposit $\mathrm{TiO}_{2}$ on the surface of a biomaterial (i.e., polymethyl methacrylate (PMMA), titanium alloy implants, and collagen) were selected for this review. PMMA was selected as an example of a polymeric ALD substrate which has wide applications in dental fields as a denture base $[6,10,11]$, titanium alloy is an example of a metallic biomaterial which is usually used in dental implant restorations [12,13], and collagen has been selected as a protein-based biomaterial with many potential applications in bone tissue engineering application [14]. The properties of these biomaterials have been reviewed elsewhere $[15,16]$.

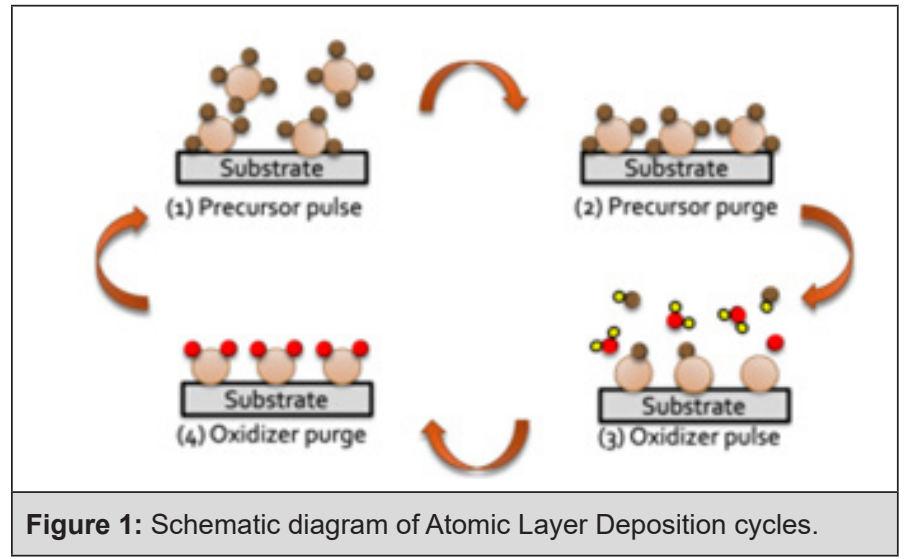

ALD of $\mathrm{TiO}_{2}$ on selected Biomaterials and Biomedical Substrates

\section{$\mathrm{TiO}_{2}$ ALD on PMMA}

PMMA has attracted much attention recently due to its biocompatibility, process ability, aesthetics, and low fabrication cost. It has been applied in dentistry as a denture base material for about 100 years [17]. However, this polymer has some potential disadvantages such as low wear-resistance, poor hardness, and water absorption. $\mathrm{TiO}_{2}$ ALD on the surface of this polymer could improve its surface wettability and mechanical properties, such as wear-resistance and surface hardness, which can enhance the performance of this polymer. Such surface improvements will decrease the microbial adherence and biofilm formation on the surface, which is essential in biomedical applications. Recent research has focused on improving the PMMA surface to overcome its limitations Huang et al. $[10,11]$ modified the surface properties of PMMA with a $\mathrm{TiO}_{2}$ coating deposited by low-temperature ALD $\left(65^{\circ} \mathrm{C}\right)$ using Tetrakis (diethylamide)titanium (TDMAT) and ozone $\left(\mathrm{O}_{3}\right)$ as the titanium and oxygen sources, respectively. Their findings indicated that the $\mathrm{TiO}_{2} \mathrm{ALD}$ coating enhanced the surface wettability and wear resistance of PMMA while maintaining its flexural properties. Moreover, a significant decrease in the number of viable attached cells of $C$. albicans on the $\mathrm{ALD} \mathrm{TiO}_{2}$-coated PMMA samples was observed compared to noncoated PMMA. In another study by Pensa [6], $\mathrm{TiO}_{2}$ thin films were deposited on PMMA substrates using thermal ALD at a rather high temperature $\left(120^{\circ} \mathrm{C}\right)$. TDMAT and $\mathrm{O}_{3}$ were used as the titanium and oxygen sources, respectively. A new ellipsometry model was presented to measure the film thickness on PMMA substrates and increased wettability and surface hardness were reported. Kääriäinen et al. [18] deposited $\mathrm{TiO}_{2}$ thin films on PMMA via ALD using TDMAT and $\mathrm{O}_{3}$ where PMMA substrates were subjected to plasma treatment. Increased wear resistance and protection of coated polymer from ultraviolet (UV) exposure were reported. Kemell et al. [19] conducted a study using ALD for the deposition of $\mathrm{TiO}_{2}$ from titanium tetrachloride $\left(\mathrm{TiCl}_{4}\right)$ and water on different polymers, including a thin film of PMMA ( 70-100 nmthick) which was spin-coated on silicon. Coated PMMA films became super hydrophilic after 20 minutes of UV illumination. Paul et al. [20] deposited different layers of metal oxides including $\mathrm{TiO}_{2}$ by PE-ALD on PMMA, on which they had deposited a layer of $\mathrm{Al}_{2} \mathrm{O}_{3}$ by thermal ALD. Their results suggest that the multi-layer films on PMMA are crack-free, adhesive, and environmentally durable.

\section{$\mathrm{TiO}_{2}$ ALD on titanium alloy implants}

Although titanium alloys have widespread applications in dental and orthopedic implants due to their biocompatibility, non-toxicity, good mechanical properties, corrosion resistance, and being bioinert, they have some disadvantages such as being hydrophobic which is not desirable for biomedical purposes [12,15]. Improving the wettability behavior of titanium alloy implants with $\mathrm{TiO}_{2} \mathrm{ALD}$ is beneficial with respect to the increased cellular attachments well as reduced microbial adherence In a study by Patel et al. [12], a thin film of $\mathrm{TiO}_{2}$ was deposited on Ti alloy (grade V) (Ti-6Al-4V) substrates using Tetrakis (diethyl amino) titanium (TDEAT) and water at $250^{\circ} \mathrm{C}$. A gradual increase in wettability was reported after each stage of treatment, including pristine, de-ionized (DI) water wash, $\mathrm{N}_{2}$ drying, sonication in methanol, ALD treatment, and post-ALD DI water wash, respectively. Besides, anatase $\mathrm{TiO}_{2}$ was obtained after 5 min $\mathrm{N}_{2}$ post-annealing at elevated temperatures $\left(600\right.$ and $\left.800^{\circ} \mathrm{C}\right)$ [12]. Liu et al. [13] conducted a study in which they functionalized the surface of $\mathrm{Ti}^{\mathrm{b}}$ by $\mathrm{TiO}_{2}$ ALD using TDMAT and water. According to their results, $\mathrm{TiO}_{2}$ coating effectively inhibited gram-positive bacteria ( $S$. aureus), gram-negative bacteria ( $E$. coli), and antibiotic-resistant bacteria (MRSA) while presenting 
desirable cellular functions. Promoted osteoblast adhesion and proliferation and suppressed fibroblast adhesion and proliferation were also reported [13]. In another study by Basiaga et al. [21], $\mathrm{TiO}_{2}$ was deposited by ALD on two grades of Ti alloys (i.e., Ti-V and Ti6Al7Nb) using $\mathrm{TiCl}_{4}$ and water. Based on their reported results, the surface corrosion resistance was improved by increasing the film thickness. Moreover, the best film adhesion was achieved at 1250 ALD cycles regardless of the substrate types. However, no significant difference in wettability and surface topography was obtained by varying the number of cycles [21].

\section{$\mathrm{TiO}_{2}$ ALD on collagen membranes}

Collagen is a biocompatible and biodegradable protein with many applications such as in bone implants, wound healing, and tissue regeneration $[14,22]$. However, it has some drawbacks such as poor mechanical properties and low fibrillar density, which may limit its stability when being implanted in the body [14,22]. A bioactive ceramic coating such as $\mathrm{TiO}_{2}$ would improve the mechanical properties of collagen and minimize its limitations. Therefore, some research studies have focused on the enhancement of collagen membranes. A comparative study between Chemical Vapor Deposition (CVD) and ALD techniques was performed [23] in which $\mathrm{TiO}_{2}$ was infiltrated using both technique son silk and collagen membranes from titanium isopropoxide and purified water as the source of titanium and oxygen, respectively. Their results revealed that compared to CVD, ALD coated biomaterials would lead to higher mechanical properties in terms of molecular fracture. Bishal et al. [24] functionalized the surface of collagen membranes using ALD at room temperature. They successfully deposited a uniform ultra-thin film of $\mathrm{TiO}_{2}$ on the surface of collagen membranes using TDMAT and $\mathrm{O}_{3}$. In a following study of their previous work. Bishal et al. [14], investigated in vitro bioactivity of $\mathrm{ALD} \mathrm{TiO}_{2}$-coated collagen membranes as a potential to promote bone regeneration in bone tissue engineering. According to their results, compared to non-coated collagen membranes, $\mathrm{ALD} \mathrm{TiO}_{2}$-coated collagens were more bioactive since they promoted more osteoblast cell growth, spreading, and cell proliferation rate as well as facilitated the nucleation of calcium phosphate or apatite formation by higher attraction of $\mathrm{Ca}$ and $\mathrm{P}$ elements when immersed in a simulated body fluid solution [14].

\section{Future Challenges}

The recent progress in functionalizing the surface of a few important biomaterials with $\mathrm{TiO}_{2}$ ALD was briefly reviewed in this article. ALD is a relatively new technique but very promising in the biomedical field. More in-vitro and in-vivo studies should be carried out to optimize the ALD conditions for different substrates and use this process as a physiologically friendly method on a large scale. Being a rather slow process is one of the main challenges with ALD, which may limit its commercial applications on a large scale, in the near future. Spatial ALD may partially overcome this issue by providing higher deposition rates, but further investigations are needed in order to establish that for commercial surface modification of biomedical substrates. Additionally, there are many types of microorganisms in the body, each of which interacts with the inserted implants separately. Thus, more microbial tests should be performed in short-term and long-term using different microorganisms. In order to better characterize the film growth on biomaterials, it is important to deposit a film with a known desired thickness on the substrates of interest. Thickness measurement of Nano films on organic substrates like PMMA using spectroscopic ellipsometry has also become increasingly important. Further experiments are still needed to obtain and enhance more accurate models.

\section{References}

1. Johnson RW, Hultqvist A, Bent SF (2014) A brief review of atomic layer deposition: from fundamentals to applications. Mater Today 17(5): 236246.

2. Mallick BC, Hsieh C T, Yin K M, Gandomi YA, Huang K T, et al. (2019) Review-On Atomic Layer Deposition: Current Progress and Future Challenges. ECS J. Solid State Sci Technol 8(4): N55-N78.

3. Profijt HB, Potts SE, van de Sanden MCM, Kessels WMM (2011) PlasmaAssisted Atomic Layer Deposition: Basics, Opportunities, and Challenges. J. Vac. Sci. Technol. A Vacuum, Surfaces, Film 29(5): 050801-1-05080126.

4. George SM (2009) Atomic layer deposition: an overview. Chem Rev 110(1): 111-131.

5. Bishal AK, Butt A, Selvaraj SK, Joshi B, Patel SB, et al. (2015) Atomic Layer Deposition in Bio-Nanotechnology: A Brief Overview. Crit Rev Biomed Eng 43(4): 255-276.

6. Pensa E (2018) Enhanced Properties of Polymethyl Methacrylate Coated with Atomic Layer Deposited Ceramic Nanofilm. University of Illinois at Chicago.

7. Jin C, Liu B, Lei Z, Sun J (2015) Structure and photoluminescence of the TiO2 films grown by atomic layer deposition using tetrakisdimethylamino titanium and ozone. Nanoscale Res Lett 10(1): 1-9

8. Alberici RM, Jardim WF (1994) Photocatalytic degradation of phenol and chlorinated phenols using AgTiO2 in a slurry reactor. Water Res. 28(8): 1845-1849.

9. Reuterg̊ardh LB, Iangphasuk M (1997) Photocatalytic decolourization of reactive azo dye: A comparison between TiO2 and CdS photocatalysis. Chemosphere 35(3): 585-596.

10. Huang S (2017) Improving Polymethyl Methacrylate Resin Using Novel Nano-Ceramic Coating. University of Illinois at Chicago

11. Darwish G, Huang S, Knoernschild K, Sukotjo C, Campbell S, et al. (2019) Improving Polymethyl Methacrylate Resin Using a Novel Titanium Dioxide Coating. Journal of Prosthodonticsc 28(9): 1011-1017.

12. Patel S, Butt a, Tao Q Royhman D, Sukotjo C, et al. (2014) Novel functionalization of Ti-V alloy and Ti-II using atomic layer deposition for improved surface wettability. Colloids Surfaces B Biointerfaces 115: 280-285.

13. Liu L, Bhatia R, Webster TJ (2017) Atomic layer deposition of nano-TiO thin films with enhanced biocompatibility and antimicrobial activity for orthopedic implants. Int J Nanomedicine 12: 8711-8723. 
14. Bishal AK, Sukotjo C, Jokisaari JR, Klie RF, Takoudis CG, et al. (2018) Enhanced Bioactivity of Collagen Fiber Functionalized with Room Temperature Atomic Layer Deposited Titania. ACS applied materials \& interfaces 10(40): 34443-34454.

15. Wang W, Liao S, Zhu Y, Liu M, Zhao Q, et al. (2015) Recent applications of nanomaterials in prosthodontics. J. Nanometer 2015: 1-11.

16. Velu R, Calais T, Jayakumar A, Raspall F (2020) a comprehensive review on bio-nanomaterials for medical implants and feasibility studies on fabrication of such implants by additive manufacturing technique. Materials (Basel) 13(1): 92.

17. George R, Prasad NS (2018) Evolution of Denture Base Materials from Past to New Era. IOSR J Dent Med Sci e-ISSN 17(11): 23-27.

18. Kääriäinen TO, Cameron DC, Tanttari M (2009) Adhesion of Ti and TiC coatings on PMMA subject to plasma treatment: Effect of intermediate layers of $\mathrm{Al}_{2} \mathrm{O}_{3}$ and $\mathrm{TiO}_{2}$ deposited by atomic layer deposition. Plasma Process Polym 6(10): 631-641.

19. Kemell M, Färm E, Ritala M, Leskelä M (2008) Surface modification of thermoplastics by atomic layer deposition of $\mathrm{Al} 2 \mathrm{O} 3$ and $\mathrm{TiO}_{2}$ thin films. Eur Polym J 44(11): 3564-3570.
20. Paul P, Pfeiffer K, Szeghalmi A (2020) Antireflection Coating on PMMA Substrates by Atomic Layer Deposition. Coatings 10(1): 64 .

21. Basiaga M, Kajzer W, Walke W, Kajzer A, Kaczmarek M, et al. (2016) Evaluation of physicochemical properties of surface modified Ti6Al4V and Ti6Al7Nb alloys used for orthopedic implants. Mater Sci Eng C 68: 851-860.

22. Marelli B, Ghezzi CE, Barralet JE, Boccaccini AR, Nazhat SN, et al. (2010) Three-dimensional mineralization of dense nanofibrillar collagen-bio glass hybrid scaffolds. Bio macromolecules11 (6): 1470-1479.

23. Lee SM, Pippel E, Knez M (2011) Metal infiltration into biomaterials by ALD and CVD: A comparative study. ChemPhysChem 12(4): 791-798

24. Bishal AK, Sukotjo C, Takoudis CG (2017) Room temperature TiO2 atomic layer deposition on collagen membrane from a titanium alkylamide precursor. J Vac Sci Technol A 35(1): 01B134-1-01B134-8. 